\title{
MRNA Surveillance
}

National Cancer Institute

\section{Source}

National Cancer Institute. mRNA Surveillance. NCI Thesaurus. Code C29645.

mRNA Surveillance works within elaborate cellular mechanisms to ensure accurate gene expression by assessing suitable mRNA quality for translation and prevents the synthesis of truncated or aberrant proteins. By nonsense-mediated decay, mRNA surveillance facilitates the detection and destruction of mRNAs that contain premature termination codons. Also, by nonstop decay, mRNA surveillance depletes mRNAs that lack in-frame termination codons. 